\title{
nature
}

biotechnology

\section{A futile cycle in cell therapy}

\author{
Should a cell therapy for heart disease with scant evidence of efficacy continue to be tested in humans?
}

\begin{abstract}
The data from PreSERVE-AMI, the largest US clinical trial of bonemarrow cells for heart-attack patients, have recently been published in Circulation Research $(\mathbf{1 2 0}, 324-331,2017)$. Although no safety issues emerged, the multicenter trial failed to meet its primary efficacy end point. PreSERVE-AMI is the latest in a litany of disappointing trial outcomes for adult stem-cell therapy in the heart. It fails to support the hypothesis that these therapies are efficacious. It does not move them further down the pathway to marketed products. Most of all, it highlights the need to re-evaluate the ethical and scientific grounds for continuing to test an ineffective procedure on patients with serious heart conditions.

In the early 2000s, several papers were published claiming that transplants of bone-marrow cells or $\mathrm{c}-\mathrm{Kit}^{+}$cardiac cells can regenerate the infarcted rodent heart. This work was quickly translated to the clinic, with reports of modest benefits in small numbers of patients. As the field progressed, it became clear that the donor cells were not forming new cardiac tissue but instead rapidly dying in large numbers or fusing with host cells. Speculation about biological mechanism turned to the activity of putative paracrine factors released by the cells.
\end{abstract}

Since the first human studies, a raft of trials-often not randomized, double-blind or well-controlled-has explored various cell populations and protocols. Whereas safety data were generally positive, efficacy results have been consistently marginal, ambiguous, or negative. A 2015 Cochrane review of 41 bone-marrow-cell trials for heart attack found no benefit for such end points as all-cause mortality, cardiovascular mortality, re-infarction, re-hospitalization, morbidity, quality of life, and left-ventricular ejection fraction (LVEF), while also noting that these conclusions were underpowered. In chronic heart disease, a 2016 Cochrane review of 38 bone-marrow-cell trials identified "low-quality evidence" of benefit for mortality, non-fatal myocardial infarction, and arrhythmias, and no benefit for "a composite incidence of mortality, non-fatal myocardial infarction, and/or rehospitalization for heart failure" and LVEF.

PreSERVE-AMI tells a similar tale. A phase 2 trial of autologous CD $34^{+}$bone-marrow cells in 161 patients with acute myocardial infarction, it found no benefit in the primary efficacy end point (improved resting myocardial perfusion) or in LVEF and infarct size. However, through a post hoc analysis of a mechanism that was not pre-specified, the authors claim to find a dose effect, with higher cell doses having more efficacy on LVEF, infarct size, and days alive and out of the hospital. In an accompanying editorial, Tompkins et al. describe this negative trial as "an important step in the field of cardiac regeneration" and corroborate the post hoc inference about cell dose, which they say "will undoubtedly lead to important new hypotheses to be tested in future trials."

But PreSERVE-AMI was not designed to detect dose effects. And correlation does not imply causation. The dose each patient received was equal to as many $\mathrm{CD} 34^{+}$cells as could be retrieved from their bone marrow. Patients who had more-abundant $\mathrm{CD} 34^{+}$cells may simply have been healthier in ways that contributed to their heart health. $P$ values in clinical trials should be interpreted with caution; they "were never intended to 'work backward' and provide novel information regarding biological mechanism of action" (JACC Basic Transl. Sci. doi:10.1016/ j.jacbts.2017.03.001, 2017).

A related strategy that has been proposed to overcome the dearth of efficacy is multiple rounds of cell transplantation. In a February Viewpoint article in Circulation Research, Editor-in-Chief Roberto Bolli argues that the "rising tide of skepticism" in the field can be addressed through a "paradigm shift" to repeated cell dosing. "Every effort should be made to translate the repeated-treatment paradigm to humans," he says, beginning with the necessary preclinical studies.

Bone-marrow and c-KIT ${ }^{+}$cells have now been administered to thousands of heart-disease patients over a decade and a half, expending institutional resources and consuming more than a billion dollars of government and private funding. Nearly all of these trials failed to meet their primary efficacy end points. Are more trials with higher or repeated doses justified? In weighing this question, one must remember what the goal is-and how to get there.

Obtaining licensure of a cell therapy requires well-informed, disciplined progress toward the generation of data that satisfy the conditions of the regulatory agency. It requires sound scientific evidence and a well-characterized mechanism of action. It requires compelling efficacy and safety data that can be replicated, and end points that the regulator deems meaningful. In the United States, for heart attack and heart failure, these end points include significant improvements in morbidity and mortality compared with standard-of-care drugs-a sophisticated arsenal of ACE inhibitors, beta blockers, aldosterone receptor antagonists, and other agents with well-understood mechanisms of action.

Cell-therapy trials for heart disease are nowhere close to having achieved this and have not been proceeding along a pathway to regulatory approval. Officials of the US Food and Drug Administration have recently described the lack of evidence of efficacy in stem-cell trials for heart failure (and other indications) as "worrisome" (NEJM 376, 1007-1009, 2017). Much more preclinical work defining a mechanism of action and demonstrating a strong rationale for exposing humans to these procedures is needed before any new trials should be contemplated.

Continuing to pour money into ineffective forms of cell therapy also diverts scarce funding from approaches in cardiac regenerative medicine that merit further investigation. These include bona fide cardiomyocytes or progenitors that may differentiate into heart muscle and defined paracrine factors that may tip the balance from fibrosis toward repair.

The mounting negative results in heart cell-therapy trials are an opportunity to reassess the trajectory of the field as a whole and develop rational priorities going forward. The groupthink that has long sustained these trials must not cloud objective appraisal of the scientific evidence. 